\title{
On the error term of the mean square formula for the Riemann zeta-function in the critical strip $3 / 4<\sigma<1$
}

\section{YuK-KAM LAU (Nancy and Hong Kong)}

1. Introduction. For $1 / 2<\sigma<1$, define

$$
E_{\sigma}(t)=\int_{0}^{t}|\zeta(\sigma+i u)|^{2} d u-\left(\zeta(2 \sigma) t+(2 \pi)^{2 \sigma-1} \frac{\zeta(2-2 \sigma)}{2-2 \sigma} t^{2-2 \sigma}\right)
$$

where $\zeta(s)$ is the Riemann zeta-function. This is an analogue of $E(t)$ for the case $\sigma=1 / 2$, which was extensively studied. Comparatively, $E_{\sigma}(t)$ is new and the following mean square formula was obtained by Matsumoto and Meurman [8] and [9] within this decade:

$$
\int_{1}^{T} E_{\sigma}(t)^{2} d t= \begin{cases}A_{1}(\sigma) T^{5 / 2-2 \sigma}+O(T) & \text { if } 1 / 2<\sigma<3 / 4 \\ A_{0} T \log T+O(T) & \text { if } \sigma=3 / 4 \\ O(T) & \text { if } 3 / 4<\sigma<1\end{cases}
$$

The $O$-term in the case $\sigma=3 / 4$ in [8] was given with a slightly weaker estimate $O(T \sqrt{\log T})$ and Lam [5] improved it to $O(T)$. Then it is natural to wonder whether the $O$-terms are sharp. This question is meaningful, especially in the last case, because it provides the information how large $E_{\sigma}(t)(3 / 4<\sigma<1)$ can be. In fact, if we denote the error term $O(T)$ by $F_{\sigma}(T)$, it is conjectured that $F_{\sigma}(T)=A(\sigma) T+o(T)$ (see [4] and [7] for more details).

Concerning the case $3 / 4<\sigma<1$, in addition to the mean square estimate in (1.1), Ivić and Matsumoto [2] proved that

$$
E_{\sigma}(t) \ll t^{4 \kappa(1-\sigma) /(1+4 \kappa(1-\sigma))} \log T
$$

where $(\kappa, \lambda)$ is an exponent pair such that $\lambda=\kappa+1 / 2$. It is the best upper bound to date. Furthermore, we found in $[6]$ that

$$
\int_{1}^{T} E_{\sigma}(t) d t=-2 \pi \zeta(2 \sigma-1) T+O(\sqrt{T}) .
$$

2000 Mathematics Subject Classification: Primary 11M06. 
This immediately implies that $F_{\sigma}(T)=\int_{1}^{T} E_{\sigma}(t)^{2} d t \gg T$ and partly answers the above-mentioned question (on whether the $O$-term in (1.1) is sharp). However, at the same time, it suggests that $E_{\sigma}(t)$ may consist of two parts: one is the constant $-2 \pi \zeta(2 \sigma-1)$ and the other has a small mean value $O(\sqrt{T})$. This was also pointed out in Matsumoto [7, p. 256]. Thus we carry out this splitting, or in other words, we consider

$E_{\sigma}^{*}(t)=\int_{0}^{t}|\zeta(\sigma+i u)|^{2} d u-\left(\zeta(2 \sigma) t+(2 \pi)^{2 \sigma-1} \frac{\zeta(2-2 \sigma)}{2-2 \sigma} t^{2-2 \sigma}-2 \pi \zeta(2 \sigma-1)\right)$.

Then we have $E_{\sigma}(t)=-2 \pi \zeta(2 \sigma-1)+E_{\sigma}^{*}(t), \int_{1}^{T} E_{\sigma}^{*}(t) d t \ll \sqrt{T}$ and $\int_{1}^{T} E_{\sigma}^{*}(t)^{2} d t \ll T$ by $(1.1)$.

Our purpose here is to study the size of the last integral $\int_{1}^{T} E_{\sigma}^{*}(t)^{2} d t$. It is interesting because if the integral is $o(T)$, then the conjecture (for the last case) is settled; otherwise, in view of its mean value, this shows that $E_{\sigma}^{*}(t)$ is highly oscillating and so is $E_{\sigma}(t)$. We find that the latter case occurs, as is anticipated (see the remark below).

TheOrem. Let $3 / 4<\sigma<1$ and $T_{0}$ be a sufficiently large constant. Then, for all $T \geq T_{0}$, we have $\int_{1}^{T} E_{\sigma}^{*}(t)^{2} d t \gg_{\sigma} T$ where the implied constant depends on $\sigma$ only.

REMARK. Let $\sigma_{a}(n)=\sum_{d \mid n} d^{a}$, and for $-1<a<0$ define

$$
\Delta_{a}(x)=\sum_{n \leq x} \sigma_{a}(n)-\zeta(1-a) x-\frac{\zeta(1+a)}{1+a} x^{1+a}+\frac{1}{2} \zeta(-a) .
$$

This is a generalization of the classical error term $\Delta(x)$ in Dirichlet's divisor problem. It is well known that there is an analogy between the error terms $\Delta(x)$ and $E(t)$. Such an analogy also appears between $\Delta_{1-2 \sigma}(x)$ and $E_{\sigma}(t)$ (or more appropriately, $E_{\sigma}^{*}(t)$ ). (This was also discussed in [4] and [7].) Indeed, there is a mean square result for $\Delta_{1-2 \sigma}(x)$, parallel to (1.1), obtained by Meurman [10] with a truncated Voronoi-type formula and delicate analysis. On the other hand, starting with another (finite) series representation, an asymptotic formula for $\int_{1}^{T} \Delta_{1-2 \sigma}(x)^{2} d x$ with $3 / 4<\sigma<1$ can be derived. This was worked out in [1] and [12] and, therefore, a similar result for $E_{\sigma}^{*}(t)$ is expected. The series representation used in [1] and [12], however, highly depends on the arithmetic nature of the divisor function and there is no counterpart of that for $E_{\sigma}(t)$ yet. (Our result is perhaps not insignificant in view of this difficulty.)

Finally, let us outline our approach. Define $G_{\sigma}(t)=\int_{1}^{t} E_{\sigma}^{*}(u) d u$. Then $G_{\sigma}(t+h)-G_{\sigma}(t)=\int_{t}^{t+h} E_{\sigma}^{*}(u) d u$ can be regarded as an approximation of $h E_{\sigma}^{*}(t)$. Thus, it leads to evaluate the mean square $\int_{T}^{2 T}\left(G_{\sigma}(t+h)-G_{\sigma}(t)\right)^{2} d t$ 
which can be treated by Jutila's argument in [3] and techniques in [9]. However, in order to make small $h$ admissible, we need a series representation of $G_{\sigma}(t)$ with a sufficiently small error term. To this end, we apply the argument in [8] to derive an averaged form of the series representation. However, we can only obtain a formula for $G_{\sigma}(2 t)-G_{\sigma}(t)$ instead of $G_{\sigma}(t)$. This needs a little extra effort to handle (see Section 4 ).

In what follows, $3 / 4<\sigma<1$ and $c_{i}(i=1,2, \ldots)$ denote unspecified positive constants which depend on $\sigma$ only.

2. Averaged formula. We start with the formula

$$
\int_{T}^{2 T} E_{\sigma}^{*}(t) d t=\left.\Sigma_{1}(t, X)\right|_{T} ^{2 T}+\left.\pi^{-1 / 2} t^{5 / 2} I(t)\right|_{T} ^{2 T}+O(1)
$$

where $X \in[A T, T]$ is not an integer with a constant $0<A<1$, and

$$
I(t)=\int_{X}^{\infty} \frac{\widetilde{\Delta}_{1-2 \sigma}(\xi) \cos (t V+2 \pi \xi U-\pi \xi+\pi / 4)}{\xi^{3} V^{2} U^{1 / 2}(U-1 / 2)^{\sigma}(U+1 / 2)^{\sigma+2}} d \xi .
$$

The function $\Sigma_{1}(t, X)$ is defined at the beginning of [6, Section 2] while $\widetilde{\Delta}_{1-2 \sigma}(\xi)$ is defined in [6, Lemma 3.2]. $U$ and $V$ are functions defined as in [6, Lemma 3.1] with $k$ replaced by $\xi$.

This formula comes from $[6,(3.3)-(3.5),(3.8)$ and (3.14)], and a simple refinement of $[6,(3.9)]$

$$
\int_{T}^{2 T} G_{4}^{*}(t) d t \ll T^{r-1 / 2} \log T \ll_{\sigma} 1 .
$$

The last upper bound relies on the fact that $r<1 / 2$ when $3 / 4<\sigma<1$. (Note that $r=-\left(4 \sigma^{2}-7 \sigma+2\right) /(4 \sigma-1)$, see [6, Lemma 3.2].)

To deal with $I(t)$, it can be observed that $I(t)$ is essentially the same as the integral $J$ in $[8$, p. 368] except that the exponent of $V$ is replaced by 2 and $\sin$ by $\cos$ in our case. We proceed with the argument in [8, Section 6$]$ by replacing $\widetilde{\Delta}_{1-2 \sigma}(\xi)$ with its Voronoi-type series. The components corresponding to $J_{2}(n, b)$ and $O\left(T^{-\sigma-7 / 4}\right)$ in $[8,(6.1)]$ are treated in the same way. Then we carry out the smoothing process described in [8, Section 7], with $X=(L+\mu)^{2}(0 \leq \mu \leq M)$ and $L=M=\sqrt{T} / 2$. It is easy to handle $\Sigma_{1}(t, X)$ but the remaining part in $I(t)$ needs a more complicated treatment, following the same lines of argument of the evaluation of $K_{n}$ in [8, p. 372]. Finally, we can obtain

$$
\int_{T}^{2 T} E_{\sigma}^{*}(t) d t=\left.\Sigma_{1}^{*}(t)\right|_{T} ^{2 T}-\left.\Sigma_{2}^{*}(t)\right|_{T} ^{2 T}+O(1) .
$$


Here, corresponding to $\Sigma_{1, \sigma}^{*}(t, L, M)$ in [8], we have

$$
\Sigma_{1}^{*}(t)=\sqrt{2}\left(\frac{t}{2 \pi}\right)^{5 / 4-\sigma} \sum_{n \leq T}(-1)^{n} w_{1}(n) \frac{\sigma_{1-2 \sigma}(n)}{n^{7 / 4-\sigma}} e_{2}(t, n) \sin f(t, n)
$$

where $w_{1}(n)=1$ if $n \leq T / 4$ and $2(1-\sqrt{n / T})$ if $T / 4<n \leq T$,

$$
\begin{aligned}
& e_{2}(t, n)=\left(1+\frac{\pi n}{2 t}\right)^{-1 / 4}\left(\sqrt{\frac{2 t}{\pi n}} \operatorname{arsinh} \sqrt{\frac{\pi n}{2 t}}\right)^{-2}, \\
& f(t, n)=2 t \operatorname{arsinh} \sqrt{\frac{\pi n}{2 t}}+\left(2 \pi n t+\pi^{2} n^{2}\right)^{1 / 2}-\frac{\pi}{4}
\end{aligned}
$$

with $\sigma_{a}(n)=\sum_{d \mid n} d^{a}$ and $\operatorname{arsinh} x=\log \left(x+\sqrt{x^{2}+1}\right)$. Moreover, following the proof of $[8,(8.2)]$ (i.e. $\left[8\right.$, p. 375]), $\Sigma_{2}^{*}(t)$ analogous to $\Sigma_{2, \sigma}^{*}(t, L, M)$ satisfies

$$
\int_{T}^{2 T} \Sigma_{2}^{*}(t)^{2} d t \ll T
$$

because $\Sigma_{2}^{*}(t)$ only differs from $\Sigma_{2, \sigma}^{*}(t, L, M)$ in the exponent of $\log (t /(2 \pi n))$ (the exponent is -2 in $\Sigma_{2}^{*}(t)$ ) and the trigonometric function $\cos g(t, n)$ $\left(\sin g(t, n)\right.$ in $\left.\Sigma_{2}^{*}(t)\right)$.

Define

$$
S(t)=\Sigma_{1}^{*}(t)-\Sigma_{2}^{*}(t) \text { and } G_{\sigma}(t)=\int_{1}^{t} E_{\sigma}^{*}(u) d u .
$$

Then from (2.1) we get

$$
G_{\sigma}(2 T)-G_{\sigma}(T)=\int_{T}^{2 T} E_{\sigma}^{*}(t) d t=S(2 T)-S(T)+\mathcal{E}
$$

where $|\mathcal{E}| \leq c_{1}$. Our next section is to study the mean square of the difference $S(t+h)-S(t)$.

3. Mean square of $S(t+h)-S(t)$. We prove the following.

LEMma 3.1. Let $B$ and $B^{\prime}$ be sufficiently large but fixed constants. Then

$$
K_{1} T h^{5-4 \sigma} \leq \int_{T}^{2 T}(S(t+h)-S(t))^{2} d t \leq K_{2} T h^{5-4 \sigma}
$$

uniformly for $B \leq h \leq \sqrt{T} / B^{\prime}$, where $K_{1}$ and $K_{2}$ are positive constants depending on $\sigma$ only. 
Proof. Using $(t+h)^{5 / 4-\sigma}-t^{5 / 4-\sigma} \ll t^{1 / 4-\sigma} h$ and

$$
e_{2}(t+h, n)-e_{2}(t, n)=\left.h \frac{\partial}{\partial t}\right|_{t=\xi} e_{2}(t, n) \ll n h t^{-2}
$$

with $\xi \in[t, t+h]$, we have, for $t \in[T, 2 T]$,

$$
\Sigma_{1}^{*}(t+h)-\Sigma_{1}^{*}(t)=\sqrt{2}\left(\frac{t}{2 \pi}\right)^{5 / 4-\sigma} \operatorname{Im} \sum_{n \leq T} a_{n} k_{n}(t) e^{i f(t, n)}+O\left(T^{-1 / 2} h\right)
$$

where

$$
\begin{aligned}
a_{n} & =(-1)^{n} w_{1}(n) \sigma_{1-2 \sigma}(n) n^{\sigma-7 / 4}, \\
k_{n}(t) & =e_{2}(t, n)(\exp (i(f(t+h, n)-f(t, n)))-1) .
\end{aligned}
$$

This yields

$$
S(t+h)-S(t)=\sqrt{2}\left(\frac{t}{2 \pi}\right)^{5 / 4-\sigma} \operatorname{Im} \sum_{n \leq T} a_{n} k_{n}(t) e^{i f(t, n)}+F(t, h)
$$

where $F(t, h)=\Sigma_{2}^{*}(t+h)-\Sigma_{2}^{*}(t)+O(1)$. In view of (2.2), it suffices to evaluate

$$
J=2 \int_{T}^{2 T}\left(\frac{t}{2 \pi}\right)^{5 / 2-2 \sigma}\left(\operatorname{Im} \sum_{n \leq T} a_{n} k_{n}(t) e^{i f(t, n)}\right)^{2} d t .
$$

Plainly,

$$
\begin{aligned}
J= & \int_{T}^{2 T}\left(\frac{t}{2 \pi}\right)^{5 / 2-2 \sigma}\left|\sum_{n \leq T} a_{n} k_{n}(t) e^{i f(t, n)}\right|^{2} d t \\
& -\operatorname{Re} \int_{T}^{2 T}\left(\frac{t}{2 \pi}\right)^{5 / 2-2 \sigma}\left(\sum_{n \leq T} a_{n} k_{n}(t) e^{i f(t, n)}\right)^{2} d t \\
= & (2 \pi)^{2 \sigma-5 / 2} \sum_{n \leq T} a_{n}^{2} \int_{T}^{2 T} t^{5 / 2-2 \sigma}\left|k_{n}(t)\right|^{2} d t+O\left(T^{5 / 2-2 \sigma}\left(\left|J_{1}\right|+\left|J_{2}\right|\right)\right)
\end{aligned}
$$

by the mean value theorem for integrals, where for some $T_{i} \in[T, 2 T](i=$ $1,2,3,4)$,

$$
\begin{aligned}
J_{1} & =\int_{T_{1}}^{T_{2}} \sum_{m \neq n \leq T} a_{m} a_{n} k_{m}(t) \overline{k_{n}(t)} e^{i(f(t, m)-f(t, n))} d t, \\
J_{2} & =\int_{T_{3}}^{T_{4}} \sum_{m, n \leq T} a_{m} a_{n} k_{m}(t) k_{n}(t) e^{i(f(t, m)+f(t, n))} d t .
\end{aligned}
$$
Then

To bound $J_{1}$, we write $k(t)=k_{m}(t) \overline{k_{n}(t)}$ and $\phi(t)=f(t, m)-f(t, n)$.

$$
J_{1}=\sum_{m \neq n \leq T} a_{m} a_{n}\left\{\left.k(t) \frac{e^{i \phi(t)}}{\phi^{\prime}(t)}\right|_{T_{1}} ^{T_{2}}-\int_{T_{1}}^{T_{2}}\left(\frac{k^{\prime}(t)}{\phi^{\prime}(t)}-k(t) \frac{\phi^{\prime \prime}(t)}{\phi^{\prime}(t)^{2}}\right) e^{i \phi(t)} d t\right\} .
$$


Noting that

$$
f(t+h, n)-f(t, n)=2 h \operatorname{arsinh} \sqrt{\frac{\pi n}{2 \xi}} \quad \text { where } \xi \in[t, t+h],
$$

we have $k_{n}(t) \ll \min (1, h \sqrt{n / t})$ as $\operatorname{arsinh} x \ll x$ for all $x \geq 0$. Applying Hilbert's inequality (see [11]) and $\min _{m \neq n}\left|\phi^{\prime}(t)\right| \gg \sqrt{n t}$, we get

$$
\begin{aligned}
\left.\sum_{m \neq n \leq T} a_{m} a_{n} k(t) \frac{e^{i \phi(t)}}{\phi^{\prime}(t)}\right|_{T_{1}} ^{T_{2}} & \ll \sum_{n \leq T} a_{n}^{2}\left|k_{n}(t)\right|^{2} \sqrt{n T} \\
& \ll T^{1 / 2} \sum_{n \leq T} \sigma_{1-2 \sigma}(n)^{2} n^{2 \sigma-3} \min (1, h \sqrt{n / T})^{2} \\
& \ll T^{2 \sigma-3 / 2} h^{4-4 \sigma} .
\end{aligned}
$$

Using the fact that $k_{n}^{\prime}(t) \ll \sqrt{n} h t^{-3 / 2}$, we have

$$
\begin{aligned}
\int_{T_{1}}^{T_{2}} \sum_{m \neq n \leq T} a_{m} a_{n} k^{\prime}(t) \frac{e^{i \phi(t)}}{\phi^{\prime}(t)} d t \\
\ll T\left(\sum_{n \leq T} a_{n}^{2}\left|k_{n}^{\prime}(t)\right|^{2} \sqrt{n T}\right)^{1 / 2}\left(\sum_{n \leq T} a_{n}^{2}\left|k_{n}(t)\right|^{2} \sqrt{n T}\right)^{1 / 2} \\
\ll T^{2 \sigma-3 / 2} h^{3-2 \sigma} .
\end{aligned}
$$

Finally, from [9, p. 379], we have

$$
\frac{\phi^{\prime \prime}(t)}{\phi^{\prime}(t)^{2}}=-\frac{1}{2 t \phi^{\prime}(t)}\left(\cosh \left(\frac{1}{2} f^{\prime}(t, m)\right)\right)^{-2}+O\left(t^{-1}\right) .
$$

Then we see that as $\cosh x \geq 1$,

$$
\begin{aligned}
\int_{T_{1}}^{T_{2}} \sum_{m \neq n \leq T} & a_{m} a_{n} k(t) \frac{\phi^{\prime \prime}(t)}{\phi^{\prime}(t)^{2}} e^{i \phi(t)} d t \\
& \ll \sum_{n \leq T} a_{n}^{2}\left|k_{n}(t)\right|^{2} \sqrt{n T}+\left(\sum_{n \leq T}\left|a_{n} k_{n}(t)\right|\right)^{2} \ll T^{2 \sigma-3 / 2} h^{4-4 \sigma} .
\end{aligned}
$$

To sum up, $J_{1} \ll T^{2 \sigma-3 / 2} h^{3-2 \sigma}$. The estimation of $J_{2}$ is easier. Taking $k(t)=k_{m}(t) k_{n}(t)$ and $\phi(t)=f^{\prime}(t, m)+f^{\prime}(t, n)$, we then have $k(t) \ll$ $\min (1, h \sqrt{n / T}), k^{\prime}(t) \ll \sqrt{n} h t^{-3 / 2}, \phi^{\prime}(t) \gg(\sqrt{m}+\sqrt{n}) / \sqrt{T}$ and $\phi^{\prime \prime}(t) \ll$ $(\sqrt{m}+\sqrt{n}) T^{-3 / 2}$. The same treatment yields $J_{2} \ll T^{2 \sigma-3 / 2} h$ and so $(3.2)$ becomes

$$
J=(2 \pi)^{2 \sigma-5 / 2} \sum_{n \leq T} a_{n}^{2} \int_{T}^{2 T} t^{5 / 2-2 \sigma}\left|k_{n}(t)\right|^{2} d t+O\left(T h^{3-2 \sigma}\right) .
$$


From this, we can deduce that

$$
\begin{aligned}
J & \leq c_{2} T^{7 / 2-2 \sigma} \sum_{n \leq T} \sigma_{1-2 \sigma}(n)^{2} n^{2 \sigma-7 / 2} \min (1, h \sqrt{n / T})^{2}+c_{3} T h^{3-2 \sigma} \\
& \leq c_{4} T h^{5-4 \sigma} .
\end{aligned}
$$

Since $x / 2 \leq \operatorname{arsinh} x \leq 2 x$ when $0 \leq x \leq 1$, we see from (3.3) that $c_{5} h \sqrt{n / T} \leq f(t+h, n)-f(t, n) \leq \pi / 2$ when $n \leq \delta T / h^{2}$ for some small constant $\delta>0$. Therefore, provided $\delta B^{\prime} \geq c_{6}(>1)$, we get

$$
\begin{aligned}
J & \geq c_{7} T^{5 / 2-2 \sigma} h^{2} \sum_{n \leq \delta T / h^{2}} \sigma_{1-2 \sigma}(n)^{2} n^{2 \sigma-5 / 2}-c_{8} T h^{3-2 \sigma} \\
& \geq\left(c_{9}-\frac{c_{8}}{B^{2-2 \sigma}}\right) T h^{5-4 \sigma} \geq c_{10} T h^{5-4 \sigma}
\end{aligned}
$$

provided $B>c_{11}$. From $(2.2)$, we see that $\int_{T}^{2 T} F(t, h)^{2} d t \ll T$, and thus from (3.1) and (3.4),

$$
\int_{T}^{2 T}(S(t+h)-S(t))^{2} d t=J+O(T+\sqrt{J T})=J+O\left(T h^{5 / 2-2 \sigma}\right) .
$$

This completes the proof of Lemma 3.1, with (3.4), (3.5) and a sufficiently large $B$.

4. Proof of Theorem. We choose an integer $R$ such that $2^{R(5-4 \sigma)} \geq$ $4 K_{2} / K_{1}$ where $K_{1}$ and $K_{2}$ are defined as in Lemma 3.1. Let

$$
h=\max \left(B,\left(12\left(c_{1} R\right)^{2} / K_{2}\right)^{1 /(5-4 \sigma)}\right)
$$

and $T$ be any sufficiently large number (in particular, $\left.T \geq 2^{R}\left(B^{\prime} h\right)^{2}\right)$. From (2.3), we have

$$
\begin{aligned}
& \left(G_{\sigma}\left(2^{r}(t+h)\right)-G_{\sigma}\left(2^{r} t\right)\right)-\left(G_{\sigma}\left(2^{r-1}(t+h)\right)-G_{\sigma}\left(2^{r-1} t\right)\right) \\
& \quad=\left(G_{\sigma}\left(2^{r}(t+h)\right)-G_{\sigma}\left(2^{r-1}(t+h)\right)\right)-\left(G_{\sigma}\left(2^{r} t\right)-G_{\sigma}\left(2^{r-1} t\right)\right) \\
& \quad=\left(S\left(2^{r}(t+h)\right)-S\left(2^{r-1}(t+h)\right)\right)-\left(S\left(2^{r} t\right)-S\left(2^{r-1} t\right)\right)+\mathcal{E}_{r}^{\prime} \\
& \quad=\left(S\left(2^{r}(t+h)\right)-S\left(2^{r} t\right)\right)-\left(S\left(2^{r-1}(t+h)\right)-S\left(2^{r-1} t\right)\right)+\mathcal{E}_{r}^{\prime}
\end{aligned}
$$

where $\left|\mathcal{E}_{r}^{\prime}\right| \leq 2 c_{1}$. Summing over $r=1, \ldots, R$, yields

$$
\begin{aligned}
\left(G_{\sigma}\left(2^{R}(t+h)\right)-G_{\sigma}\left(2^{R} t\right)\right)-\left(G_{\sigma}(t+h)-G_{\sigma}(t)\right) & \\
= & \left(S\left(2^{R}(t+h)\right)-S\left(2^{R} t\right)\right)-(S(t+h)-S(t))+\sum_{r=1}^{R} \mathcal{E}_{r}^{\prime} .
\end{aligned}
$$

Now, we square both sides and integrate over $[T, 2 T]$. Using the inequality $2\left(a^{2}+b^{2}\right) \geq(a-b)^{2} \geq a^{2} / 2-b^{2}$, we infer that 


$$
\begin{aligned}
& \int_{T}^{2 T}\left(G_{\sigma}\left(2^{R}(t+h)\right)-G_{\sigma}\left(2^{R} t\right)\right)^{2} d t+\int_{T}^{2 T}\left(G_{\sigma}(t+h)-G_{\sigma}(t)\right)^{2} d t \\
& \geq 4^{-1} \int_{T}^{2 T}\left(\left(S\left(2^{R}(t+h)\right)-S\left(2^{R} t\right)\right)-(S(t+h)-S(t))\right)^{2} d t-\frac{T}{2}\left|\sum_{r=1}^{R} \mathcal{E}_{r}^{\prime}\right|^{2} \\
& \geq 4^{-1}\left(\frac{1}{2^{R+1}} \int_{2^{R} T}^{2^{R+1} T}\left(S\left(t+2^{R} h\right)-S(t)\right)^{2} d t-\int_{T}^{2 T}(S(t+h)-S(t))^{2} d t\right) \\
& \quad-2\left(c_{1} R\right)^{2} T \\
& \geq \frac{K_{2}}{4} T h^{5-4 \sigma}-2\left(c_{1} R\right)^{2} T \geq\left(c_{1} R\right)^{2} T
\end{aligned}
$$

by Lemma 3.1 with our choices of $R$ and $h$. Using the Cauchy-Schwarz inequality, we see that

$$
\int_{T}^{2 T}\left(G_{\sigma}(t+h)-G_{\sigma}(t)\right)^{2} d t=\int_{T}^{2 T}\left|\int_{t}^{t+h} E_{\sigma}^{*}(u) d u\right|^{2} d t \leq h^{2} \int_{T}^{2 T+h} E_{\sigma}^{*}(u)^{2} d u .
$$

As $R$ and $h$ are fixed constants, we conclude that $\int_{T}^{2^{R+2} T} E_{\sigma}^{*}(u)^{2} d u \gg T$ and hence the result.

Acknowledgements. The author would like to express his sincere gratitude to Professor Kohji Matsumoto for his valuable comments and helpful discussions.

\section{References}

[1] S. Chowla, Contributions to the analytic theory of numbers, Math. Z. 35 (1932), 279-299.

[2] A. Ivić and K. Matsumoto, On the error term in the mean square formula for the Riemann zeta-function in the critical strip, Monatsh. Math. 121 (1996), 213-229.

[3] M. Jutila, On the divisor problem for short intervals, Ann. Univ. Turku. Ser. A I 186 (1984), 23-30.

[4] I. Kiuchi and K. Matsumoto, The resemblance of the behaviour of the remainder terms $E_{\sigma}(t), \Delta_{1-2 \sigma}(x)$ and $R(\sigma+i t)$, in: Sieve Methods, Exponential Sums, and their Applications in Number Theory, G. R. H. Greaves et al. (eds.), London Math. Soc. Lecture Note Ser. 237, Cambridge Univ. Press, 1997, 255-273.

[5] K.-Y. Lam, Some results on the mean values of certain error terms in analytic number theory, M. Philos. Thesis, Univ. of Hong Kong, 1997.

[6] K.-Y. Lau, A study of the mean value of the error term in the mean square formula of the Riemann zeta-function in the critical strip $3 / 4 \leq \sigma<1$, manuscript. 
[7] K. Matsumoto, Recent developments in the mean square theory of the Riemann zeta and other zeta-functions, in: Number Theory, Trends Math., Birkhäuser, Basel, 2000, 241-286.

[8] K. Matsumoto and T. Meurman, The mean square of the Riemann zeta-function in the critical strip III, Acta Arith. 64 (1993), 357-382.

[9] - - - The mean square of the Riemann zeta-function in the critical strip II, ibid. 68 (1994), 369-382.

[10] T. Meurman, The mean square of the error term in a generalization of Dirichlet's divisor problem, ibid. 74 (1996), 351-364.

[11] H. Montgomery and R. C. Vaughan, Hilbert's inequality, J. London Math. Soc. (2) 8 (1974), 73-82.

[12] N. Yanagisawa, An asymptotic formula for a certain mean value in a divisor problem, J. Number Theory 73 (1998), 339-358.

Institut Élie Cartan

Université Henri Poincaré (Nancy 1)

54506 Vandœuvre-lès-Nancy Cedex, France
Current address:

Department of Mathematics The University of Hong Kong Pokfulam Road, Hong Kong E-mail: yklau@maths.hku.hk

Received on 16.10.2000

and in revised form on 12.7.2001 